\title{
OF DEFECTS IN MUSICAL INSTRUMENTS AND THEIR VALUE
}

\author{
By J. A. FULLER-MAITLAND
}

$I^{1}$ $T$ is possible that many who have taken an interest in the material of music have indulged at one time or another in dreams of some perfect instrument that should combine the merits and characters of all existing instruments, while avoiding all their defects. A piano capable of sustaining a tone as long as the player might desire, a horn that should possess all the notes of the chromatic scale without sacrificing its individuality, an organ that should give a faithful imitation of every orchestral instrument without loss of its characteristic "diapason" tone, a violin whose notes could be excited by an endless bow, so that no interval could be detected by the sharpest ear at the change of the bow's direction, -these are some of the ideals that have engaged the attention of so many enthusiasts, that it seems worth while to point out what value there may be in the inherent defects of the various instruments, and in how large a measure their character is due to these very shortcomings.

Music has never been a perfect art; even without reference to man-made instruments, the central problem of intonation is insoluble, as the Greeks knew well. For if a circle of perfect fifths be constructed, and the intervals C-G, G-D, D-A, and so on, be tuned perfectly (or the equivalent obtained by alternately descending a perfect fourth and rising a perfect fifth), the $C$ that will be arrived at is at a different pitch from the starting-point by a small interval known as the Pythagorean comma, amounting to about a quarter of a semitone. The history of music would be a very different thing from what it is if this tiny error could have been done away with by some miraculous agency. As it is, scientific and practical musicians in various ages have tried different expedients in order to conceal its existence, and to obtain the benefits of having all keys perfectly in tune. As every one knows, the violinist must learn to make slight compromises in many places in order to produce the effect of being in tune during changes of key; the older keyboard instruments dealt only with the simpler keys, and made these perfectly in tune, banishing the essential error of the comma into keys with many flats or 
sharps in the signature, keys that were virtually never used. The system of equal temperament gave to the world Bach's immortal masterpiece, the "Wohltemperirtes Clavier" which was written to encourage the adoption by clavichord-tuners of the compromise to which we are all so well accustomed in the present day. For in musicians' lives, whatever their medium of expression, the piano has played such a part that there are comparatively few people who really feel its fifths to be too flat and its major thirds too sharp. There are here and there to be found choirs capable of realizing the fact that voices can be tuned without submission to a piano, and perfect intervals can sometimes be heard even now in unaccompanied music. Occasionally string quartet players attain to just intonation, but they, like the choral singers, must yield to the compromise whenever they are associated with the piano. One or two elaborate inventions have been made at various times for obtaining pure intonation from a keyboard. The "quarter-tone" keys on the old organ in the Temple Church, General PerronetThompson's organ with its forty pipes to the octave, and Mr. Shohe Tanaka's easily grasped arrangement of divided keys, are specimens of these attempts, but none has ever come into general use, by reason of the complications of the keyboard, and the heavy demands on musicianship entailed in the choice of the true intervals, even supposing sufficient manual dexterity to have been reached.

Coming to the various instruments of music, there is hardly one which can be called absolutely perfect. Characteristic qualities of wind instruments, such as the shrillness of the piccolo, the sweetness of the flute, the poignancy of the oboe, or the snarl of the bassoon, are so unchangeable that the study of one of them must involve a degree of monotony that would be hard to face; even the clarinet, though it has a far greater range of expression, and greater variety of tone-qualities than the rest of the "woodwind," would clog the ear of most students long before proficiency was attained. But even the clarinet, producing as it does only those "upper partials" or "overtones" that are unevenly numbered in the complete series, cannot be said to be ideally perfect. The horn-tone, when the "natural" or "Waldhorn" is used, is of exquisite beauty, but its available notes are limited to the harmonic series, and every enlargement of its scope, whether by the use of crooks for the various keys, or by other means, brings about a certain deterioration of its individuality. The trumpet, in like manner, loses a good deal of its beauty when keys are applied to it, and it finally degenerates into the cornet-à-pistons which can 
perform anything, but is satisfactory in nothing. Perhaps the trombone is the happiest of the brass section of the orchestra, for the quality of its tone is not affected by the slide, which allows all keys to be employed, with slight and very valuable differences of effect in their employment. The kettledrums, again, are as nearly perfect as can be wished, and the only defect here is that the player has only two hands, whereas he should have at least enough to manipulate seven drums.

Continuing the survey down the staves of an orchestral score, we come next to the violin, which holds, as it deserves to hold, the supreme position among the instruments. Its structure has been enormously improved since the distant day when it was first developed from the rebec, the fidel, and the crwth. The alteration in the sound-post is mainly responsible for the greatly increased resonance of tone, and the natural process of the gradual seasoning of the wood, and, perhaps, the influence of the varnish used by the Italian makers, have given the violin its supremacy. And, be it noted, these are all improvements along the natural line of its development, not efforts to make the instrument do something it never set out to do. Some later experiments in other directions have been so miserably ineffectual that they have been practically laughed out of court. They are almost entirely limited to the effort to produce a continuous tone, and disguising the moment at which the player changes the direction of his bow. A rosined wheel was devised in fairly ancient times, and the king of instruments was degraded to the "hurdy-gurdy" or "vielle" which survived long enough to be seen about the streets of London as late as the middle of the XIXth century. At international exhibitions some of the most obstinately recurrent inventions have the same object in view, but even if they could obtain a footing, it would be found that continuity had only been reached by the sacrifice of articulation. A continuous stream of melody without points of climax, and without recognizable phrases distinct from each other, is without life. It is very much the same with certain other inventions, familiar to those who haunt exhibitions, which have for their object the prolongation of the pianoforte tone, whether by slight hammer-strokes rapidly repeated, or by other means.

The lower "strings" of the orchestra come very near the level reached in the violin, and the violoncello might almost have usurped the throne, but for the presence of what is called the "Wolf," a phenomenon common to all stringed instruments, but most noticeable in the violoncello. Every enclosed, or partially- 
enclosed space is a "resonating box" for some note or other, and when that note is sounded the framework of the box is set in "sympathetic vibration." The body of the violoncello is such a box, and when the note to which it is in sympathetic resonance is sounded, the whole structure vibrates so strongly that the strings cannot be held quite taut, so that the quality of the tone suffers; it is a curious paradox that the note to which the whole being of the instrument is most sympathetic, is the one note it cannot play perfectly. Whether any efforts have been made to counteract this defect, does not greatly concern us, since none has succeeded.

The key'ed instruments are not better off than the others. The modern organ is a perfect chamber of horrors, each of them perpetrated in the name of improvements, and in the search for a greater, if still mechanical, expressiveness. The organ-makers of old, desiring a less pure and more incisive tone than the simple pipes could give, added "mixture-stops," supplying the upper notes of the harmonic series by artificial means; and while stops with that name, or with the title "twelfth" are commonly found in the present day, the curious "sesquialtera" and "cornet" stops have died a natural death. Yet, used with discretion, they are none of them unpleasing, and the "cornet voluntaries" of the English XVIIIth century composers are very dull if none but $8 \mathrm{ft}$. and $4 \mathrm{ft}$. stops are used. Wonderfully close imitations of flute, oboe, and clarinet tones, were devised at various times, and all such things were legitimate enough; the "swell pedal," whatever its method of construction, never quite allows the crescendo to continue long enough, but squanders so much of the increase of tone at the beginning, that there is nothing left for the end. But it is an indispensable adjunct to the resources of the organ, and does allow musicians (as well as other people) to suggest an almost emotional phrasing. It is quite another thing with the dreadful family of stops which, under such names as "Unda maris," "Voix célestes," "Vox Angelica," "Vox Humana," "Vox Gregis" or the like, strive to imitate some of the worst faults of popular singers, and only succeed in copying the bleating of an asthmatic sheep. The least offensive form of the appendage is that which produces a tremulous effect by the tuning of two sets of pipes slightly at variance with each other, so that "beats" are produced. The worst is of course that in which the quivering is simulated by a series of regularly recurring flappings of a kind of fan.

There is not much to be said of the other keyed instruments that are fed with wind; none has ever claimed to be a perfect instrument, and the merit they aim at, that of cheapness, is 
certainly attained. From the concertina and melodeon up to the harmonium and American organ, the inherently strident tone cannot be got rid of, and although the principle of the "expression stop" on the harmonium is legitimate enough and very ingenious, it is so difficult to master that few players attempt its use.

The history of the stringed instruments with keys, from the virginal to the pianoforte, is marked by many and various attempts to improve them in different ways, and by a few efforts to get rid of the defect they all have in common, that of the evanescent quality of the tone, which, as a compensation, and unlike all other instruments except the harp, sounds on for a short time, though for a short time only, after the process of sounding is over. The simple plucking of a stretched string without modification of any kind, which is typical of the virginal, was improved into the perfected harpsichord, such as was made in London at the close of the XVIIIth century; the processes by which variety of tone and power was given, were, one and all, legitimate, and the instrument has few real defects apart from those arising from its poverty of volume and the curious nasal sound produced by the twanging of the plectra. When the dulcimer was improved into the clavichord by the substitution of keys for the hammers held in the player's hand, as in the Hungarian cimbalom and the still surviving English dulcimer, the first step towards the pianoforte was taken, and despite its tiny amount of tone, the instrument, for which Bach wrote the "Chromatic Fantasia" as well as the "48," remains one of the most purely artistic in the world for its range of expression within the narrow limits of its power. Before the pianoforte could be developed from it, the peculiar beauty of the clavichord must be sacrificed, and the device given up by which the finger acting on the tangent remains in virtual contact with the string, and so makes it possible to execute the "bebung," a tremolo that is quite unmechanical. Down to the time when the first attempt was made to prolong the pianoforte tone at pleasure, as above mentioned, the improvements in its structure were mainly such as were perfectly allowable (I am not now thinking of the "Turkish" or "Janissary" pedal, and some similar contrivances which were only fashionable for a short time): for most of the improvements were based on a recognition of the effects that are natural to the instrument and peculiar to it. Though directed to increasing the volume of tone, they seldom lost sight of the fact that the sound could still be detached, as it were, from the exciting medium, and the beautiful device of the sustaining pedal allowed the sympathetic vibrations of the unstruck strings to be used to add to the sonority of the 
whole. It is this precious fact, that strings do sound sympathetically, that has promoted the harp from its old office of a domestic vehicle for the display of a shapely arm, to an honoured place in the orchestra, where the sympathetic resonance of its strings adds to the general sonority, and the instrument seems to gain an amount of power altogether unsuspected by the older composers. Here again attempts have been made to lessen the player's difficulties, and one of the attempts, the double-action pedal of Erard has made an enormous and legitimate difference in the harp's artistic position; another, the ingenious method of stringing employed in the "chromatic harp", only manages to dispense with the elaborate pedals by the sacrifice of the curious glissando effects on arpeggios of the diminished seventh and other strange chords.

It seems fairly certain that in almost all instruments of music there do exist some inherent defects which make for individuality and which are really merits in disguise. In case after case, we have seen how their removal, so far from being an advantage, has had a tendency to reduce the differences of quality to one dead level of colourless tone.

What, then, is the "moral" of all this? Shall we welcome all inherent defects as if they were merits, and sit contented with inferior workmanship, rejecting all suggestions for improvements? Shall we value performers according to their lack of skill in their art, and pay the largest fees to pianists who play the greatest number of wrong notes? Already there are signs that in some quarters faults have been worth money, as when a conscientious contralto singer was rejected by a concert-agent in London with the words, "You see, if you only had a nice break in your voice like Mme. . . . . . we might do something for you, but as it is, your voice is too even."

We must surely discriminate between the kind of improvement that aims at copying the excellences of some different medium of music, and the kind that proceeds along the line of natural development of the instrument's resources. To reject both classes alike would be to stultify all the improvements that have brought the various instruments to their present state, and the logical conclusion would be we should never allow a child to be taught a scale for fear of its losing its musical innocence. If an increase of pianoforte sonority can only be attained by a sacrifice of tone-quality, - as was the case with the pianos of the latter part of the XIXth century, when defence against the onslaughts of herculean pianists seemed to be necessary, and when the bass strings were made to give out sounds scarcely recognizable as notes, - then let that 
Of Defects in Musical Instruments and Their Value 97

increase of volume wait till some material can be found for the bass strings which will let them still be musically beautiful. Let the oboe remain an oboe, the clarinet a clarinet, and above all the organ an organ; for no blending of tone can compensate for loss of the characteristic organ quality, nor can fancy stops atone for inferiority in the texture of organ diapasons. 